\title{
Retrospective comparison of craniotomy and decompressive craniectomy for surgical evacuation of nontraumatic, supratentorial intracerebral hemorrhage
}

\author{
Seth B. Hayes, M.D., Ronald J. Benveniste, M.D., Ph.D., Jacques J. Morcos, M.D., \\ Mohammad A. Aziz-Sultan, M.D., and Mohamed Samy Elhammady, M.D. \\ Department of Neurological Surgery, University of Miami Miller School of Medicine, Miami, Florida
}

\begin{abstract}
Surgical evacuation of nontraumatic, supratentorial intracerebral hemorrhage (SICH) is uncommonly performed, and outcomes are generally poor. On the basis of published experimental data and the authors' anecdotal observations, a retrospective chart review study was performed to test the hypothesis that large decompressive craniectomies (DCs), compared with craniotomies, would improve clinical outcomes after surgical evacuation of SICH. For patients with putaminal SICH, DC was associated with a statistically significant improvement in midline shift, compared with craniotomy. Decompressive craniectomies also resulted in a strong trend toward decreased likelihood of poor neurological outcome (modified Rankin Scale score $>3$ ). For patients with lobar SICH, no associations were found between DC or craniotomy and clinical outcomes. For patients selected to undergo surgical evacuation of putaminal SICH, a DC in addition to surgical evacuation of the hematoma might improve outcome. (http://thejns.org/doi/abs/10.3171/2013.2.FOCUS12422)
\end{abstract}

\section{KEY WordS - intracerebral hemorrhage - craniotomy • decompressive craniectomy}

$\mathrm{N}$ ONTRAUMATIC SICH represents $10 \%-15 \%$ of all strokes and often carries a poor prognosis..$^{4,16,23}$ Although aggressive surgical intervention is recommended for patients with large or symptomatic hypertensive cerebellar hemorrhages, ${ }^{13}$ most patients with SICH are unlikely to benefit from surgical evacuation. The most widely cited and relevant study to date is the STICH, which failed to show benefit from surgical evacuation for patients with SICH, whose treating physicians were unsure whether surgery would be beneficial. ${ }^{10}$ However, the design of the STICH (only patients in "treatment equipoise" were randomized) and post hoc data analysis suggest that some patients with SICH should undergo surgery. $7,10,11$

After the results of the STICH were published, surgical evacuation of SICH became an uncommon procedure, reserved for a small subset of patients, at our insti-

\footnotetext{
Abbreviations used in this paper: $\mathrm{DC}=$ decompressive craniectomy; GCS = Glasgow Coma Scale; $\mathrm{mRS}=$ modified Rankin Scale; SICH $=$ supratentorial intracerebral hemorrhage; STICH = Surgical Trial in Intracerebral Haemorrhage.
}

tution (University of Miami Miller School of Medicine). In general, we consider surgical intervention for patients who are relatively young; who have a large, symptomatic $\mathrm{SICH}$; who have some preservation of brainstem function; and who can undergo surgery very soon (within a few hours at most) after their initial neurological deterioration. Under these circumstances, we operate on deep basal ganglia hemorrhages and on more superficial lobar hemorrhages. Unfortunately, even among this select group of patients, clinical outcomes remain poor. Thus, the neurosurgical community continues to seek ways to improve outcomes for the subset of patients who meet the criteria for surgical evacuation.

Clinical and laboratory research has demonstrated that $\mathrm{SICH}$ is followed by injury to the surrounding brain; this secondary injury might result from various mechanisms including excitotoxicity, direct toxicity of the hematoma, and inflammation., ${ }^{1,2}$ These various forms of secondary injury cause cytotoxic edema, which can cause increased mass effect and tissue damage. On the basis of these studies and our own anecdotal observations of the occasional need for further surgery after evacuation of 


\section{S. B. Hayes et al.}

SICH via small craniotomies, we hypothesized that surgical treatment for SICH might be optimized by larger DCs, analogous to the treatment of mass effect caused by cytotoxic edema in the setting of head trauma and ischemic stroke. To test this hypothesis, we performed a retrospective chart review to compare outcomes after evacuation of SICH with craniotomy and replacement of the bone flap and after evacuation of SICH with DC.

\section{Methods}

\section{Study Design}

After obtaining institutional review board approval for the study, we used department billing records and surgeons' case logs to retrospectively identify study participants. We identified 51 patients at the University of Miami Miller School of Medicine with SICH who had undergone surgical decompression during 2004-2012; these dates were selected because comprehensive electronic medical records and digital radiology images were available during this period. We recognized that because of small sample size and poor clinical outcomes, the study would be underpowered to detect differences in clinically meaningful measures such as survival and neurologic outcomes. We therefore decided to also study a surrogate measure of the technical adequacy of surgical decompression, namely, the degree of midline shift. We recognized that, in clinical terms, patients with hypertensive putaminal hemorrhage represented a rather homogeneous and distinctive subpopulation compared with the very heterogeneous population of patients who had lobar hemorrhage that required surgery. We therefore studied these 2 patient populations separately.

\section{Surgical Procedures}

For each case, the goal was evacuation of the hemorrhage, usually assisted by microscopy. Endoscopic procedures and stereotactic guided hematoma aspiration were not performed during the study period. Patients who underwent DC only, without evacuation of the hematoma, were not included in the study. At the time of the procedure, the operating surgeon decided, in a nonrandomized fashion, whether to perform a large or small craniotomy and whether to replace the bone flap or leave it off.

For all cases, putaminal hemorrhages were approached by using the operating microscope, and a large or small frontotemporal craniotomy or DC was performed as described. ${ }^{5}$ The small craniotomies were generally standard pterional craniotomies intended to facilitate exposure of the sylvian fissure; the DCs were generally trauma-type flaps intended to maximize decompression of the cerebral hemisphere and usually extended to the floor of the middle fossa to maximize decompression of the tentorial incisura and brainstem. In some cases, the sylvian fissure was split under the microscope to allow less traumatic access to the insula and deep hematoma; however, for most cases, the hematoma was drained by making a small corticotomy in the superior temporal gyrus or other noneloquent location. At the discretion of the operating surgeon, intraoperative ultrasonography was used for some cases.

\section{Data Analysis}

According to review of preoperative CT images, patients were classified as having had putaminal or lobar hemorrhage. Review of medical records and $\mathrm{CT}$ images provided preoperative and intraoperative clinical data and postoperative outcome data. Given the small numbers of patients, we decided to use the combined measure of death or severe disability (defined as mRS Score 4-5) as our primary clinical outcome. Secondary outcomes included death within 30 days and need for reoperation. Given the generally poor neurologic outcomes and the small size of our patient population and consequent low statistical power of our study to detect differences in meaningful clinical outcomes, we decided to also measure preoperative and postoperative midline shift. We believed that midline shift would indicate the technical success of surgery and quantify the relief of mass effect resulting from hematoma and secondary tissue injury. To determine the maximal midline shift, we reviewed radiologists' reports; when the midline shift was not specified in a report, it was measured at the foramen of Monro by using digital measuring tools available on our viewing software. Midline shift was quantified preoperatively and immediately postoperatively; if multiple postoperative CT images were available, the maximum postoperative midline shift was also measured. Categorical variables were compared by using the 2-tailed Fisher exact test; continuous variables were compared by using the MannWhitney U-test.

\section{Results}

\section{Patients With Putaminal SICH}

Of the 26 patients who underwent surgery for putaminal SICH, DCs were performed in 10 and craniotomies were performed in 16 (1 patient in this latter group had a small pterional craniotomy, and the bone flap was left off at the end of the surgery because of intraoperative brain swelling). Preoperative clinical data and postoperative outcomes for patients with putaminal SICH are shown in Table 1. Patients with putaminal SICH who underwent DC were more likely than those who underwent craniotomy to have a preoperative GCS score less than 8 (p $=0.019$ ); also among patients who underwent DC, there were trends, although not statistically significant, toward younger age and greater likelihood of right-sided SICH. The 2 groups did not differ with respect to any other preoperative variables studied, including the degree of preoperative midline shift. Differences in clinical outcomes including poor outcome (death or mRS score of 4-5), death within 30 days, and mean mRS score were not statistically significant between the 2 groups. Among patients who underwent DC, there was a trend toward lower likelihood of poor outcome ( $\mathrm{p}=0.14)$; of note, the only 2 patients with good clinical outcomes were in the DC group, and the only 2 patients who required reoperation were in the craniotomy group (although this finding was not statistically significant). However, the degree of midline shift on initial postoperative CT image and the maximal degree of postoperative midline shift were significantly less for 


\section{Craniotomy versus decompressive craniectomy for SICH}

TABLE 1: Clinical variables and outcomes among patients who underwent surgery for putaminal SICH*

\begin{tabular}{|c|c|c|c|}
\hline Variable & Decompressive Craniectomy $(n=10)$ & Craniotomy $(n=16)$ & p Value \\
\hline age (yrs; mean \pm SD) & $50 \pm 12$ & $58 \pm 11$ & $0.096 \dagger$ \\
\hline $\operatorname{sex}(M / F)$ & $4: 6$ & $9: 7$ & $0.688 \ddagger$ \\
\hline GCS Score $<8$ & $10 / 10(100)$ & $7 / 14(50)$ & $0.019 \ddagger$ \\
\hline surgery w/in 24 hrs & $6 / 9(67)$ & $12 / 15(80)$ & $0.635 \ddagger$ \\
\hline $\mathrm{rt} / \mathrm{lt}$ side & $5: 2$ & $3: 7$ & $0.051 \neq$ \\
\hline hematoma volume (ml) & $57 \pm 6$ & $70 \pm 12$ & $0.136 \dagger$ \\
\hline intraop brain relaxation & $2 / 7(29)$ & $5 / 7(71)$ & $0.286 \ddagger$ \\
\hline $\begin{array}{l}\text { poor clinical outcome (death or } \\
\text { mRS Score 4-5) }\end{array}$ & $7 / 9(78)$ & $14 / 14(100)$ & $0.142 \ddagger$ \\
\hline improved motor function & $1 / 10(10)$ & $2 / 13(15)$ & $1.00 \ddagger$ \\
\hline improved language/cognition & $6 / 10(60)$ & $4 / 15(27)$ & $0.122 \ddagger$ \\
\hline \multicolumn{4}{|l|}{ midline shift (mm; mean $\pm \mathrm{SD}$ ) } \\
\hline preop & $9.5 \pm 4.3$ & $8.4 \pm 3.5$ & $0.802 \dagger$ \\
\hline postop & $4.1 \pm 2.2$ & $7.5 \pm 2.8$ & $0.016 \dagger$ \\
\hline $\max$ & $4.2 \pm 2.3$ & $8.4 \pm 2.6$ & $0.006 \dagger$ \\
\hline reoperation & $0 / 10(0)$ & $2 / 16(13)$ & $0.508 \ddagger$ \\
\hline death w/in 30 days & $3 / 9(33)$ & $4 / 15(27)$ & $1.00 \ddagger$ \\
\hline
\end{tabular}

patients who underwent DC than for patients who underwent craniotomy ( $\mathrm{p}=0.016$ and $\mathrm{p}=0.006$, respectively). In addition, the average degree of midline shift decreased after surgery only in patients who underwent DC and not in patients who underwent craniotomy.

Among patients in the DC group, 4 eventually underwent cranioplasty, and 1 of these patients required a third surgery to remove the cranioplasty because of infection. Another patient required placement of a ventriculoperitoneal shunt for hydrocephalus.

\section{Patients With Lobar SICH}

Of the 25 patients who underwent surgery for lobar $\mathrm{SICH}$, DCs were performed in 8 and craniotomies were performed in 17. All patients in the DC group underwent transcortical hematoma evacuation (Table 2). The DC and craniotomy groups were less well matched among patients with lobar SICH than among patients with putaminal SICH. Patients with lobar SICH who underwent DC had greater preoperative midline shift $(p=0.022)$ and were much more likely to have right-sided SICH (p $=0.011$ ) than were patients who underwent craniotomy. Among patients in the DC group, there were trends, although not statistically significant, toward younger age, female sex, and larger hematoma volume. The likelihoods of poor clinical outcome, death within 30 days, and reoperation did not differ between the 2 groups, nor did postoperative midline shift. There were no statistically significant differences, or strong trends toward differences, in any of the clinical or radiographic outcomes between patients with lobar SICH who had undergone DC and those who had undergone craniotomy.

Two patients required reoperation for wound infec- tion. Postoperative hydrocephalus did not develop in either of these patients.

\section{Discussion}

Since publication of the STICH, surgical evacuation of SICH has become less common at many centers, including our own. ${ }^{9}$ However, our own experiences, as well as post hoc data analysis from the STICH and other published studies, indicate that there is probably a small subset of SICH patients who will benefit from surgical evacuation. ${ }^{3,6,8,10,15,19}$ Ongoing research, including the STICH 2 , is intended to help clinicians preoperatively identify these patients. ${ }^{7,11}$ A complementary area of research aims to identify the optimal surgical techniques for patients who are selected to undergo surgical evacuation of SICH. Most of the available literature is focused on minimal access techniques, such as stereotactic-guided hematoma aspiration and endoscopic clot evacuation, both of which are intended to minimize the disruption of intact brain tissue superficial to the hematoma. ${ }^{2,12,14,17,20}$ We believe that although these minimal access techniques might have a role in treating deeper, thalamic, or even brainstem SICH, there are technical advantages to open surgical approaches for putaminal and lobar SICH. The use of open techniques and a microscope allows for more rapid clot decompression and facilitates meticulous hemostasis at the walls of the hematoma cavity. In addition, our data suggest that at least for deep basal ganglia hemorrhages, open decompression with DC might optimize outcomes.

We identified only 1 other published study in the English language that compared craniotomy and craniectomy among patients selected to undergo open surgical evacua- 
TABLE 2: Clinical variables and outcomes among patients who underwent surgery for lobar SICH*

\begin{tabular}{|c|c|c|c|}
\hline Variable & Decompressive Craniectomy $(n=8)$ & Craniotomy $(n=17)$ & p Value \\
\hline age (yrs; mean \pm SD) & $46 \pm 12$ & $57 \pm 15$ & $0.091 \dagger$ \\
\hline $\operatorname{sex}(M / F)$ & $3: 5$ & $13: 4$ & $0.072 \ddagger$ \\
\hline GCS Score $<8$ & $6 / 8(75)$ & $6 / 15(40)$ & $0.193 \ddagger$ \\
\hline surgery w/in 24 hrs & $7 / 8(88)$ & 15/17 (88) & $1.0 \ddagger$ \\
\hline $\mathrm{rt} / \mathrm{t}$ side & $7: 1$ & $5: 12$ & $0.011 \ddagger$ \\
\hline hematoma volume (ml) & $150 \pm 92$ & $75 \pm 24$ & $0.087 \dagger$ \\
\hline intraop brain relaxation & $4 / 6(67)$ & $3 / 5(60)$ & $1.0 \ddagger$ \\
\hline $\begin{array}{l}\text { poor clinical outcome (death or mRS Score } \\
4-5 \text { ) }\end{array}$ & $4 / 8(50)$ & $8 / 17(47)$ & $1.0 \ddagger$ \\
\hline improved motor function & $6 / 8(75)$ & $6 / 13(46)$ & $0.366 \ddagger$ \\
\hline improved language/cognition & $6 / 8(75)$ & $8 / 15(53)$ & $0.400 \ddagger$ \\
\hline \multicolumn{4}{|l|}{ midline shift (mm; mean \pm SD) } \\
\hline preop & $12 \pm 3$ & $8 \pm 3$ & $0.022 \dagger$ \\
\hline postop & $5 \pm 6$ & $5 \pm 3$ & $0.542 \dagger$ \\
\hline $\max$ & $5.3 \pm 6.1$ & $6.3 \pm 5.4$ & $0.327 \dagger$ \\
\hline reoperation & 0 & 0 & $1.0 \ddagger$ \\
\hline death w/in 30 days & $2 / 8(25)$ & $6 / 17(35)$ & $1.0 \ddagger$ \\
\hline
\end{tabular}

tion of SICH. Shimamura et al. ${ }^{18}$ performed a single-center retrospective analysis of 25 patients with $\mathrm{SICH}$ who underwent either DC or clot removal. In contrast to our observations, they concluded that DC did not decrease midline shift, decrease hematoma volume, or improve outcome. However, that study was smaller than ours; only 5 of 25 patients were allocated to the DC group. Additionally, in the Shimamura et al. study, data from lobar and deep putaminal SICH were combined. On the basis of our observations and the post hoc data analysis from the STICH trial, we suggest that for purposes of surgical planning these $\mathrm{SICH}$ subtypes should be considered as 2 distinct groups. We cannot confidently explain why more aggressive decompression seems to be beneficial for putaminal SICH. It is possible that putaminal SICH causes more tissue damage as a result of involvement of the more friable deep gray matter, as opposed to lobar hemorrhages, which affect white matter to a greater degree; also, deeper hemorrhages with 360 degrees of exposure to surrounding brain might adversely affect a larger surface area, as opposed to lobar hemorrhages that breach the cortical surface. In addition, surgery for putaminal SICH often requires more tissue dissection than surgery for lobar SICH.

We recognize several potentially serious limitations of our study, but despite these limitations we suggest that useful clinical conclusions can be drawn from our data. The first limitation is that our study is retrospective. Treatment was determined at the time of surgery by the treating surgeon, based on entirely subjective criteria. The retrospective nature of this study is reflected in the differences in preoperative variables between the treatment groups. We recognize this limitation but point out that the preoperative differences between patients with putaminal SICH who underwent DC (worse GCS score and greater likelihood of right-sided hemorrhage compared with those who underwent craniotomy) were unlikely to affect the most important difference in outcome, namely postoperative midline shift. Despite the worse preoperative GCS scores, there was a trend toward improved outcomes among patients who underwent DC rather than craniotomy. The second limitation is that our small sample sizes and the poor prognosis associated with SICH result in low statistical power to detect differences in the most clinically significant outcome measures, such as death and poor functional status after surgery. We attempted to address this limitation in part by studying a surrogate measure of the technical outcome of surgery (postoperative midline shift), which showed a robust, statistically significant improvement in patients with putaminal SICH who underwent DC rather than craniotomy. We suggest that the trend toward improved neurologic outcomes with DC, and our limited observation that reoperation was performed only for the group of patients who underwent craniotomy, is consistent with our observations about this surrogate outcome.

\section{Conclusions}

We suggest that DC combined with hematoma evacuation improves outcomes in patients who undergo surgery for putaminal SICH and should be performed in patients selected for this uncommon surgical procedure. We propose that for patients with lobar SICH who undergo surgery, the decision whether to perform craniotomy or craniectomy should be individualized to the patient, considering factors such as the hematoma size, degree of midline shift, and intraoperative brain swelling. 


\section{Craniotomy versus decompressive craniectomy for SICH}

\section{Disclosure}

Dr. Aziz-Sultan is a proctor and course instructor for ev3.

Author contributions to the study and manuscript preparation include the following. Conception and design: Benveniste. Acquisition of data: all authors. Analysis and interpretation of data: Benveniste, Hayes, Morcos. Drafting the article: Hayes. Critically revising the article: Benveniste, Hayes, Morcos. Reviewed submitted version of manuscript: all authors. Approved the final version of the manuscript on behalf of all authors: Benveniste. Study supervision: Benveniste.

\section{References}

1. Aronowski J, Zhao X: Molecular pathophysiology of cerebral hemorrhage: secondary brain injury. Stroke 42:1781-1786, 2011

2. Auer LM, Deinsberger W, Niederkorn K, Gell G, Kleinert R, Schneider G, et al: Endoscopic surgery versus medical treatment for spontaneous intracerebral hematoma: a randomized study. J Neurosurg 70:530-535, 1989

3. Batjer HH, Reisch JS, Allen BC, Plaizier LJ, Su CJ: Failure of surgery to improve outcome in hypertensive putaminal hemorrhage. A prospective randomized trial. Arch Neurol 47: 1103-1106, 1990

4. Caplan LR: Intracerebral haemorrhage. Lancet 339:656-658, 1992

5. Farhat H, Kretschmer T, Morcos JJ: Nonlesional spontaneous intracerebral hemorrhage, in Winn HR (ed): Youmans Neurological Surgery, ed 6. Philadelphia: Elsevier Saunders, 2011, Vol 4, pp 3706-3729

6. Fernandes HM, Gregson B, Siddique S, Mendelow AD: Surgery in intracerebral hemorrhage. The uncertainty continues. Stroke 31:2511-2516, 2000

7. Gregson BA, Broderick JP, Auer LM, Batjer H, Chen XC, Juvela $S$, et al: Individual patient data subgroup meta-analysis of surgery for spontaneous supratentorial intracerebral hemorrhage. Stroke 43:1496-1504, 2012

8. Juvela S, Heiskanen O, Poranen A, Valtonen S, Kuurne T, Kaste M, et al: The treatment of spontaneous intracerebral hemorrhage. A prospective randomized trial of surgical and conservative treatment. J Neurosurg 70:755-758, 1989

9. Kirkman MA, Mahattanakul W, Gregson BA, Mendelow AD: The effect of the results of the STICH trial on the management of spontaneous supratentorial intracerebral haemorrhage in Newcastle. Br J Neurosurg 22:739-747, 2008

10. Mendelow AD, Gregson BA, Fernandes HM, Murray GD, Teasdale GM, Hope DT, et al: Early surgery versus initial conservative treatment in patients with spontaneous supratentorial intracerebral haematomas in the International Surgical Trial in Intracerebral Haemorrhage (STICH): a randomised trial. Lancet 365:387-397, 2005

11. Mendelow AD, Gregson BA, Mitchell PM, Murray GD, Rowan EN, Gholkar AR: Surgical trial in lobar intracerebral haemorrhage (STICH II) protocol. Trials 12:124, 2011
12. Morgan T, Zuccarello M, Narayan R, Keyl P, Lane K, Hanley D: Preliminary findings of the minimally-invasive surgery plus rtPA for intracerebral hemorrhage evacuation (MISTIE) clinical trial. Acta Neurochir Suppl 105:147-151, 2008

13. Morgenstern LB, Hemphill JC III, Anderson C, Becker K, Broderick JP, Connolly ES Jr, et al: Guidelines for the management of spontaneous intracerebral hemorrhage: a guideline for healthcare professionals from the American Heart Association/American Stroke Association. Stroke 41:21082129,2010

14. Newell DW, Shah MM, Wilcox R, Hansmann DR, Melnychuk E, Muschelli J, et al: Minimally invasive evacuation of spontaneous intracerebral hemorrhage using sonothrombolysis. Clinical article. J Neurosurg 115:592-601, 2011

15. Prasad K, Mendelow AD, Gregson B: Surgery for primary supratentorial intracerebral haemorrhage. Cochrane Database Syst Rev (4):CD000200, 2008

16. Rincon F, Mayer SA: Intracerebral hemorrhage: getting ready for effective treatments. Curr Opin Neurol 23:59-64, 2010

17. Schaller C, Rohde V, Meyer B, Hassler W: Stereotactic puncture and lysis of spontaneous intracerebral hemorrhage using recombinant tissue-plasminogen activator. Neurosurgery 36: 328-335, 1995

18. Shimamura N, Munakata A, Naraoka M, Nakano T, Ohkuma $\mathrm{H}$ : Decompressive hemi-craniectomy is not necessary to rescue supratentorial hypertensive intracerebral hemorrhage patients: consecutive single-center experience. Acta Neurochir Suppl 111:415-419, 2011

19. Steiner T, Vincent C, Morris S, Davis S, Vallejo-Torres L, Christensen MC: Neurosurgical outcomes after intracerebral hemorrhage: results of the Factor Seven for Acute Hemorrhagic Stroke Trial (FAST). J Stroke Cerebrovasc Dis 20:287-294, 2011

20. Teernstra OP, Evers SM, Lodder J, Leffers P, Franke CL, Blaauw G: Stereotactic treatment of intracerebral hematoma by means of a plasminogen activator: a multicenter randomized controlled trial (SICHPA). Stroke 34:968-974, 2003

21. UK-TIA Study Group: United Kingdom transient ischaemic attack (UK-TIA) aspirin trial: interim results. Br Med J (Clin Res Ed) 296:316-320, 1988

22. Wang J: Preclinical and clinical research on inflammation after intracerebral hemorrhage. Prog Neurobiol 92:463-477, 2010

23. Woo D, Broderick JP: Spontaneous intracerebral hemorrhage: epidemiology and clinical presentation. Neurosurg Clin $\mathbf{N}$ Am 13:265-279, 2002

Manuscript submitted December 27, 2012.

Accepted February 13, 2013.

Please include this information when citing this paper: DOI: 10.3171/2013.2.FOCUS12422.

Address correspondence to: Ronald J. Benveniste, M.D., Ph.D., Department of Neurological Surgery, University of Miami Miller School of Medicine, 1095 NW 14th Terrace (D4-6), Miami, Florida 33136. email: rbenveniste@med.miami.edu. 